\title{
Maximizing the Total Resolution of Graphs
}

\author{
Evmorfia N. Argyriou, Michael A. Bekos, and Antonios Symvonis \\ School of Applied Mathematical \& Physical Sciences, \\ National Technical University of Athens, Greece \\ \{fargyriou, mikebekos, symvonis\}@math.ntua.gr
}

\begin{abstract}
A major factor affecting the readability of a graph drawing is its resolution. In the graph drawing literature, the resolution of a drawing is either measured based on the angles formed by consecutive edges incident to a common node (angular resolution) or by the angles formed at edge crossings (crossing resolution). In this paper, we evaluate both by introducing the notion of "total resolution", that is, the minimum of the angular and crossing resolution. To the best of our knowledge, this is the first time where the problem of maximizing the total resolution of a drawing is studied.

The main contribution of the paper consists of drawings of asymptotically optimal total resolution for complete graphs (circular drawings) and for complete bipartite graphs (2-layered drawings). In addition, we present and experimentally evaluate a force-directed based algorithm that constructs drawings of large total resolution.
\end{abstract}

\section{Introduction}

There exist several criteria that have been used to judge the quality of a graph drawing 414. An undesired property that may negatively influence the readability of a graph drawing is the presence of edges that are too close to each other, especially if these edges are adjacent. Thus, maximizing the angles among incident edges becomes an important aesthetic criterion, since there is some correlation between the involved angles and the visual distinctiveness of the edges. On the other hand, recent cognitive experiments by Huang et al. 1213] indicate that the negative impact of an edge crossing on the human understanding of a graph drawing is eliminated in the case where the crossing angle is greater than 70 degrees. This motivates us to study a new graph drawing scenario in which both angular and crossing resolution are taken into account in order to produce a straight-line drawing of a given graph. Formally, the term angular resolution denotes the smallest angle formed by two adjacent edges incident to a common node, whereas the term crossing resolution refers to the smallest angle formed by a pair of crossing edges. The angular resolution maximization problem has been extensively studied by the graph drawing community over the last few decades [3910 11 15 16]. On the other hand, the crossing resolution maximization is a relatively new problem [16]7. To the best of our knowledge, this is the first attempt, where both angular and crossing resolution are combined to produce drawings.

U. Brandes and S. Cornelsen (Eds.): GD 2010, LNCS 6502, pp. 6267 2011.

(C) Springer-Verlag Berlin Heidelberg 2011 


\section{Drawings with Optimal Total Resolution for Complete and Complete Bipartite Graphs}

In this section, we define the total resolution of a drawing and we present drawings of asymptotically optimal total resolution for complete graphs (circular drawings) and complete bipartite graphs (2-layered drawings).

Definition 1. The total resolution of a drawing is defined as the minimum of its angular and crossing resolution.

We first consider the case of complete graphs $K_{n}, n \geq 3$. Our aim is to construct a circular drawing of $K_{n}$ of maximum total resolution. Our approach is constructive and common when dealing with complete graphs. A similar one has been given by Formann et al. [9] for obtaining optimal drawings of complete graphs, in terms of angular resolution. Consider a circle $\mathcal{C}$ of radius $r_{c}>0$ centered at $(0,0)$ and circumscribe a regular $n$-polygon $\mathcal{Q}$ on $\mathcal{C}$. The nodes of $K_{n}$ coincide with the vertices of $\mathcal{Q}$. The construction supports the following Theorem. For a detailed proof, refer to [2].

Theorem 1. A complete graph $K_{n}$ admits a drawing of total resolution $\Theta\left(\frac{1}{n}\right)$.

Obviously, the bound of the total resolution of a complete bipartite graph can be implied by the bound of the complete graph. However, if the nodes of the graph must have integer coordinates, i.e., we restrict ourselves on grid drawings, few results are known regarding the area needed of such a drawing. An upper bound of $O\left(n^{3}\right)$ area can be implied by [3]. This motivates us to separately study the class of complete bipartite graph, since we can drastically improve this bound. Note that tradeoffs between (angular or crossing) resolution and area have been studied by the graph drawing community, in the past [15 16].

Let $K_{m, n}=\left(V_{1} \cup V_{2}, E\right)$ be a complete bipartite graph, where $V_{1}=\left\{u_{1}^{1}, \ldots\right.$, $\left.u_{m}^{1}\right\}, V_{2}=\left\{u_{1}^{2}, \ldots, u_{n}^{2}\right\}$ and $E=V_{1} \times V_{2}$. Let $\mathcal{R}=A B \Gamma \Delta$ be a square whose top and bottom sides coincide with $\mathcal{L}_{1}$ and $\mathcal{L}_{2}$, respectively. The nodes of $V_{1}$ $\left(V_{2}\right)$ reside along side $\Gamma \Delta(A B)$. Let $\ell_{1}, \ldots, \ell_{m}$, be a bundle of semi-lines, each of which emanates from vertex $B$ and crosses side $\Gamma \Delta$ of $\mathcal{R}$, so that the angle formed by $B \Gamma$ and semi-line $\ell_{i}$ equals to $\frac{(i-1) \cdot \widehat{\Delta B \Gamma}}{m-1}$, for each $i=1, \ldots, m$. These semi-lines split angle $\widehat{\triangle B \Gamma}$ into $m-1$ angles, each of which is equal to $\frac{\pi}{4 \cdot(m-1)}$. Then, we place node $u_{i}^{1}$ at the intersection of semi-line $l_{i}$ and $\Gamma \Delta$, for each $i=1, \ldots, m$. We denote by $a_{i}$ the horizontal distance between two consecutive nodes $u_{i}^{1}$ and $u_{i+1}^{1}, i=1, \ldots, m-1$. Symmetrically, we define the position of the nodes of $V_{2}$ along side $A B$ of $\mathcal{R}$. We conclude by the following Theorem. For a detailed proof refer to [2].

Theorem 2. A complete bipartite graph $K_{m, n}$ admits a 2-layered drawing of total resolution $\Theta\left(\frac{1}{\max \{m, n\}}\right)$.

Say that the nodes of the graph must have integer coordinates. Then, assuming that $\mathcal{L}_{1}$ and $\mathcal{L}_{2}$ coincide with two horizontal grid lines, we can slightly move 
each node of $V_{1}$ and $V_{2}$ to the rightmost grid-point to its left. We can prove that there are not two nodes sharing the same grid point, assuming that $a_{1}$ is greater than one grid unit. Since no node moves more than one unit of length, the total resolution is not asymptotically affected. Regarding the computation of the area occupied by the drawing, we can further prove that it supports the following Theorem. The reader is referred to [2], for a detailed proof.

Theorem 3. A complete bipartite graph $K_{m, n}$ admits a 2-layered grid drawing of $\Theta\left(\frac{1}{\max \{m, n\}}\right)$ total resolution and $O\left(\max \left\{m^{2}, n^{2}\right\}\right)$ area.

\section{A Force Directed Algorithm}

We present a force-directed algorithm that given a reasonably nice initial drawing, results in a drawing of high total resolution. Our algorithm uses the attractive forces of the classical force-directed algorithm of Eades 8 and some additional forces exerted to the nodes of the graph, that tend to maximize the total resolution of the drawing.

Before we proceed with the description of our algorithm, we present some notation that is heavily used in the remainder. Given a drawing $\Gamma(G)$ of $G$, we denote by $p_{u}=\left(x_{u}, y_{u}\right)$ the position of node $u \in V$ on the plane. The unit length vector from $p_{u}$ to $p_{v}$ is denoted, by $\overrightarrow{p_{u} p_{v}}$, where $u, v \in V$. The degree of node $u \in V$ is denoted by $d(u)$. Let also $d(G)=\max _{u \in V} d(u)$ be the degree of the graph. Given a pair of points $q_{1}, q_{2} \in \mathbb{R}^{2}$, with a slight abuse of notation, we denote by $\left\|q_{1}-q_{2}\right\|$ the Euclidean distance between $q_{1}$ and $q_{2}$. We refer to the line segment defined by $q_{1}$ and $q_{2}$ as $\overline{q_{1} q_{2}}$. Let $\vec{\alpha}$ and $\vec{\gamma}$ be two vectors. The vector which bisects the angle between $\vec{\alpha}$ and $\vec{\gamma}$ is $\frac{\vec{\alpha}}{\|\vec{\alpha}\|}+\frac{\vec{\gamma}}{\|\vec{\gamma}\|}$. We denote by $\operatorname{Bsc}(\vec{\alpha}, \vec{\gamma})$ the corresponding unit length vector. Given a vector $\vec{\beta}$, we refer to the unit length vector which is perpendicular to $\vec{\beta}$ and precedes it in the clockwise direction, as $\operatorname{Perp}(\vec{\beta})$.

Let $e=(u, v)$ and $e^{\prime}=\left(u^{\prime}, v^{\prime}\right)$ be two crossing edge and let $p_{c}$ be their intersection point. Let also $\theta_{v v^{\prime}}, \theta_{v^{\prime} u}, \theta_{u u^{\prime}}$ and $\theta_{u^{\prime} v}$ be the angles formed by the intersection of $e$ and $e^{\prime}$ at $p_{c}$, as illustrated in Fig 1a Then, our preference for right-angle crossings can be captured by spring forces (see Fig 1a), and, by the angles $\theta_{v v^{\prime}}$ and $\theta_{v^{\prime} u}$ formed at the crossing (see Fig $1 \mathrm{~b}$ ). The exact formulas of these force are:

$$
\begin{gathered}
\mathcal{F}_{\text {spring }}^{\text {cros }}\left(p_{v}, p_{v^{\prime}}\right)=C_{\text {spring }}^{\text {cros }} \cdot \log \frac{\left\|p_{v}-p_{v^{\prime}}\right\|}{\ell_{\text {spring }}^{v v^{\prime}}} \cdot \overrightarrow{p_{v} p_{v^{\prime}}} \\
\mathcal{F}_{\text {angle }}^{\text {cros }}\left(p_{v}, p_{v^{\prime}}\right)=C_{\text {angle }}^{\text {cros }} \cdot \operatorname{sign}\left(\theta_{v v^{\prime}}-\frac{\pi}{2}\right) \cdot f\left(\theta_{v v^{\prime}}\right) \cdot \operatorname{Perp}\left(\operatorname{Bsc}\left(\overrightarrow{p_{c} p_{v}}, \overrightarrow{p_{c} p_{v^{\prime}}}\right)\right)
\end{gathered}
$$

where the constants $C_{\text {spring }}^{\text {cros }}$ and $C_{\text {angle }}^{\text {cros }}$ are used to control the stiffness of the springs and the strength of the force, respectively, $\ell_{\text {spring }}^{v v^{\prime}}$, which corresponds to the natural length of the spring, is equal to $\sqrt{\left\|p_{c}-p_{v}\right\|^{2}+\left\|p_{c}-p_{v}^{\prime}\right\|^{2}}$ and 


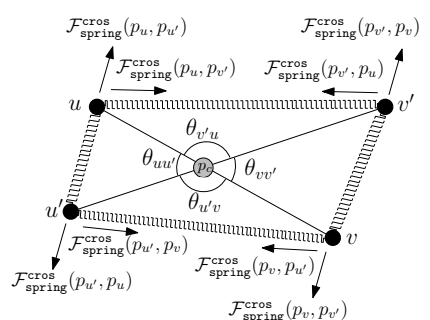

(a) Spring forces

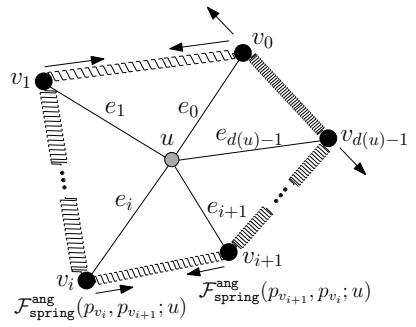

(c) Spring forces

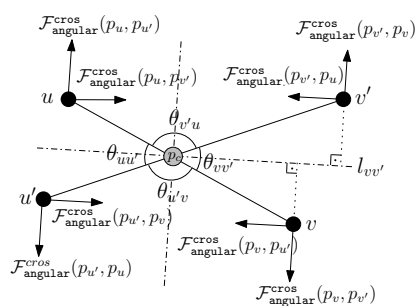

(b) Angle based forces

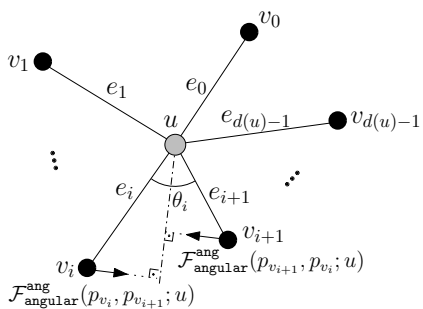

(d) Angle based forces

Fig. 1. Forces applied on nodes in order to maximize the total resolution

$f: \mathbb{R} \rightarrow \mathbb{R}$ is a function so that $f(\theta)=\left|\frac{\pi}{2}-\theta\right| / \theta$. The remaining forces of Fig 1a and $1 \mathrm{~b}$ are defined similarly.

Let $u$ be a node incident to edges $e_{0}=\left(u, v_{0}\right), \ldots e_{d(u)-1}=\left(u, v_{d(u)-1}\right)$. Assume that $e_{0}, e_{1}, \ldots, e_{d(u)-1}$ are consecutive in the counter-clockwise order around $u$ in the drawing of the graph. Let $\theta_{i}$ be the angle formed by $e_{i}$ and $e_{(i+1) \bmod (d(u))}$, measured in counter-clockwise direction from $e_{i}$ to $e_{(i+1) \bmod (d(u))}$. Then, our preference for angles equal to $2 \pi / d(u)$ can be captured by spring forces (see Fig 1c), and, by the angles $\theta_{i}, i=0,1, \ldots, d(u)-1$ (see Fig 1d). The exact formulas of these force are:

$$
\begin{aligned}
& \mathcal{F}_{\text {spring }}^{\text {angular }}\left(p_{v_{i}}, p_{v_{(i+1) \bmod (d(u))}} ; u\right)=C_{\text {spring }}^{\text {angular }} \cdot \log \frac{\left\|p_{v_{i}}-p_{v_{(i+1) \bmod (d(u))}}\right\|}{\ell_{\text {spring }}^{i}} . \\
& \overrightarrow{p_{v_{i}} p_{v_{(i+1) \bmod (d(u))}}} \\
& \mathcal{F}_{\text {angle }}^{\text {angular }}\left(p_{v_{i}}, p_{v_{(i+1) \bmod (d(u))}} ; u\right)=C_{\text {angle }}^{\text {angular }} \cdot \operatorname{sign}\left(\theta_{i}-\frac{2 \pi}{d(u)}\right) \cdot g\left(\theta_{i} ; u\right) . \\
& \operatorname{Perp}\left(\operatorname{Bsc}\left(\overrightarrow{p_{u} p_{v_{i}}}, \overrightarrow{p_{u} p_{v_{(i+1) \bmod (d(u))}}}\right)\right)
\end{aligned}
$$

where the quantities $C_{\text {spring }}^{\text {angular }}$ and $C_{\text {angle }}^{\text {angular }}$ are constants which captures the stiffness of the spring and the strength of the force, respectively, $g: \mathbb{R} \times V \rightarrow \mathbb{R}$ is a function so that $g(\theta ; u)=\frac{\left|\frac{2 \pi}{d(u)}-\theta\right|}{\theta}$ and $l_{\text {spring, }}^{i}$, which corresponds to the natural length of each spring, is equal to:

$$
\sqrt{\left\|e_{i}\right\|^{2}+\left\|e_{(i+1) \bmod (d(u))}\right\|^{2}-2 \cdot\left\|e_{i}\right\| \cdot\left\|e_{(i+1) \bmod (d(u))}\right\| \cdot \cos (2 \pi / d(u))}
$$




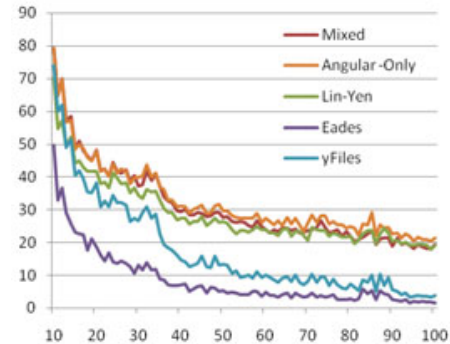

(a) Angular resolution results

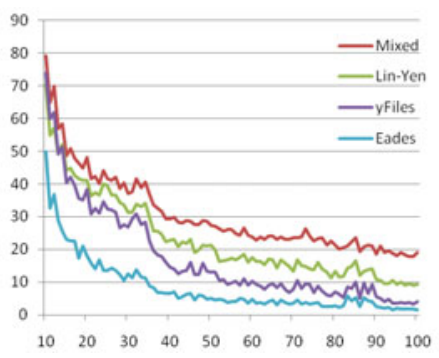

(c) Total resolution results

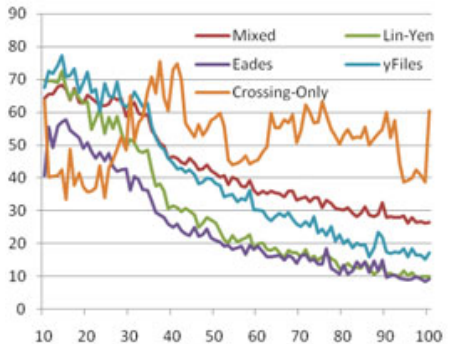

(b) Crossing resolution results

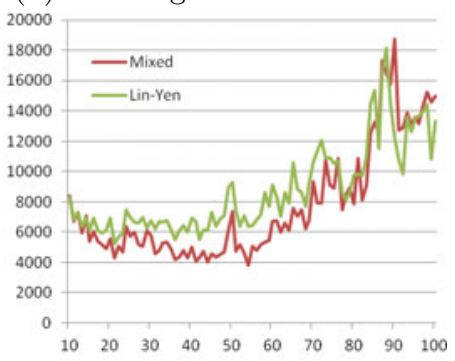

(d) Running time results

Fig. 2. A visual presentation of our experimental results. The $X$-axis indicates the number of the nodes of the graph. In Fig.(a)-(c) the $Y$-axis corresponds to the resolution measured in degrees, whereas in Fig.(d) to the running time measured in milliseconds.

Note that by setting zero values to the constants $C_{\text {spring }}^{\text {cros }}, C_{\text {angle }}^{\text {cros }}$ or $C_{\text {spring }}^{\text {angular }}$, $C_{\text {angle }}^{\text {angular }}$, our algorithm can be configured to maximize the angular, or the crossing resolution only, respectively. Regarding the time complexity, each iteration of our algorithm takes $O\left(E^{2}+V d(G) \log d(G)\right)$ time and can be further improved to $O\left(K+E \log ^{2} E / \log \log E+V d(G) \log d(G)\right)$ time per iteration, using standard techniques from computation geometry.

In the following, we present the results of the experimental evaluation of our algorithm. Apart from our algorithm, we have also implemented the algorithms of Eades 8 and Lin and Yen [15. The implementations are in Java using yFiles library (www.yworks.com). The experiment was performed on a Linux machine with $2.00 \mathrm{GHz} \mathrm{CPU}$ and 2GB RAM using the Rome graphs obtained from graphdrawing.org. The experiment was performed as follows. Each Rome graph was laid out using the SmartOrganic layouter of yFiles. This layout was the input of all algorithms. If both the angular and the crossing resolution between two consecutive iterations of each algorithm were not improved more that 0.001 degrees, we assumed that the algorithm has converged. The maximum number of iterations was set to 100.000. Our algorithm is evaluated as (a) Crossing-Only, (b) Angular-Only and (c) Mixed. Fig 2 illustrates the results of the experimental evaluation. For a more detailed analysis refer to [2]. 


\section{Conclusions}

We introduced and studied the total resolution maximization problem. Of course, our work leaves several open problems. It would be interesting to try to identify other classes of graphs that admit optimal drawings. Even the case of planar graphs is of interest, as by allowing some edges to cross (say at large angles), we may improve the angular resolution and therefore the total resolution.

\section{References}

1. Angelini, P., Cittadini, L., di Battista, G., Didimo, W., Frati, F., Kaufmann, M., Symvonis, A.: On the perspectives opened by right angle crossing drawings. In: Eppstein, D., Gansner, E.R. (eds.) GD 2009. LNCS, vol. 5849, pp. 21-32. Springer, Heidelberg (2010)

2. Argyriou, E.N., Bekos, M.A., Symvonis, A.: Maximizing the total resolution of graphs. Technical Report arXiv:/106871 (2010)

3. Bárány, I., Tokushige, N.: The minimum area of convex lattice n-gons. Combinatorica 24(2), 171-185 (2004)

4. Battista, G.D., Eades, P., Tamassia, R., Tollis, I.G.: Algorithms for drawing graphs: an annotated bibliography. Comput. Geom. 4, 235-282 (1994)

5. Cheng, C.C., Duncanyz, C.A., Goodrichz, M.T., Kobourovz, S.G.: Drawing planar graphs with circular arcs. Discrete and Comp. Geometry 25, 117-126 (1999)

6. Di Giacomo, E., Didimo, W., Liotta, G., Meijer, H.: Area, curve complexity, and crossing resolution of non-planar graph drawings. In: Eppstein, D., Gansner, E.R. (eds.) GD 2009. LNCS, vol. 5849, pp. 15-20. Springer, Heidelberg (2010)

7. Didimo, W., Eades, P., Liotta, G.: Drawing graphs with right angle crossings. In: Dehne, F., Gavrilova, M., Sack, J.-R., Tóth, C.D. (eds.) WADS 2009. LNCS, vol. 5664, pp. 206-217. Springer, Heidelberg (2009)

8. Eades, P.: A heuristic for graph drawing. Congressus Numerantium (42), 149-160 (1984)

9. Formann, M., Hagerup, T., Haralambides, J., Kaufmann, M., Leighton, F., Symvonis, A., Welzl, E., Woeginger, G.: Drawing graphs in the plane with high resolution. SIAM J. Comput. 22(5), 1035-1052 (1993)

10. Garg, A., Tamassia, R.: Planar drawings and angular resolution: Algorithms and bounds. In: Proc. 2nd Annu. Eur. Sympos. Alg., pp. 12-23 (1994)

11. Gutwenger, C., Mutzel, P.: Planar polyline drawings with good angular resolution. In: Whitesides, S.H. (ed.) GD 1998. LNCS, vol. 1547, pp. 167-182. Springer, Heidelberg (1999)

12. Huang, W.: Using eye tracking to investigate graph layout effects. In: Proc. AsiaPacific Symp. on Visualization, pp. 97-100 (2007)

13. Huang, W., Hong, S.-H., Eades, P.: Effects of crossing angles. In: Proc. of the Pacific Visualization Symp., pp. 41-46 (2008)

14. Kaufmann, M., Wagner, D. (eds.): Drawing Graphs: Methods and Models. LNCS, vol. 2025. Springer, Heidelberg (2001)

15. Lin, C.-C., Yen, H.-C.: A new force-directed graph drawing method based on edgeedge repulsion. In: Proc. of the 9th Int. Conf. on Inf. Vis., pp. 329-334. IEEE, Los Alamitos (2005)

16. Malitz, S.M., Papakostas, A.: On the angular resolution of planar graphs. In: STOC, pp. 527-538 (1992) 